\title{
The Supremum Norm of the Discrepancy Function: Recent Results and Connections
}

\author{
Dmitriy Bilyk and Michael Lacey
}

\begin{abstract}
A great challenge in the analysis of the discrepancy function $D_{N}$ is to obtain universal lower bounds on the $L^{\infty}$ norm of $D_{N}$ in dimensions $d \geq 3$. It follows from the $L^{2}$ bound of Klaus Roth that $\left\|D_{N}\right\|_{\infty} \geq\left\|D_{N}\right\|_{2} \gtrsim(\log N)^{(d-1) / 2}$. It is conjectured that the $L^{\infty}$ bound is significantly larger, but the only definitive result is that of Wolfgang Schmidt in dimension $d=2$. Partial improvements of the Roth exponent $(d-1) / 2$ in higher dimensions have been established by the authors and Armen Vagharshakyan. We survey these results, the underlying methods, and some of their connections to other subjects in probability, approximation theory, and analysis.
\end{abstract}

\section{Introduction}

We survey recent results on the sup-norm of the discrepancy function. For integers $d \geq 2$, and $N \geq 1$, let $\mathscr{P}_{N} \subset[0,1]^{d}$ be a finite point set with cardinality $\sharp \mathscr{P}_{N}=N$. Define the associated discrepancy function by

$$
D_{N}(x)=\sharp\left(\mathscr{P}_{N} \cap[0, x)\right)-N|[0, x)|,
$$

where $x=\left(x_{1}, \ldots, x_{d}\right)$ and $[0, x)=\prod_{j=1}^{d}\left[0, x_{j}\right)$ is a rectangle with antipodal corners at 0 and $x$, and $|\cdot|$ stands for the $d$-dimensional Lebesgue measure. The dependence upon the selection of points $\mathscr{P}_{N}$ is suppressed, as we are mostly interested in bounds that are universal in $\mathscr{P}_{N}$. The discrepancy function $D_{N}$ measures equidistribution of

Dmitriy Bilyk

School of Mathematics, University of Minnesota, Minneapolis MN 55455, USA, e-mail: dbilyk@math.umn.edu

Michael Lacey

School of Mathematics, Georgia Institute of Technology, Atlanta GA 30332, USA, e-mail: lacey@math.gatech.edu 
$\mathscr{P}_{N}$ : a set of points is well-distributed if this function is small in some appropriate function space.

It is a basic fact of the theory of irregularities of distribution that relevant norms of this function in dimensions 2 and higher must tend to infinity as $N$ grows. The classic results are due to Roth [21] in the case of the $L^{2}$ norm and Schmidt [23] for $L^{p}, 1<p<2$.

Theorem 1. For $1<p<\infty$ and any collection of points $\mathscr{P}_{N} \subset[0,1]^{d}$, we have

$$
\left\|D_{N}\right\|_{p} \gtrsim(\log N)^{(d-1) / 2} .
$$

Moreover, we have the endpoint estimate

$$
\left\|D_{N}\right\|_{L(\log L)^{(d-1) / 2}} \gtrsim(\log N)^{(d-1) / 2} .
$$

The symbol " $\gtrsim$ " in this paper stands for "greater than a constant multiple of", and the implied constant may depend on the dimension, the function space, but not on the configuration $\mathscr{P}_{N}$ or the number of points $N$. The Orlicz space notation, such as $L(\log L)^{\beta}$, is explained in the next section, see (6).

We should mention that there exist sets $\mathscr{P}_{N}$ that meet the $L^{p}$ bounds (1) in all dimensions. This remarkable fact is established by beautiful and quite non-trivial constructions of the point distributions $\mathscr{P}_{N}$. We refer to the reader to one of the very good references [2, 11, 12] on the subject for more information on this important complement to the subject of this note.

While the previous theorem is quite adequate for $L^{p}, 1<p<\infty$, the endpoint cases of $L^{\infty}$ and $L^{1}$ are not amenable to the same techniques. Indeed, the extremal $L^{\infty}$ bound should be larger than the average $L^{2}$ norm. In dimension $d=2$ the endpoint estimates are known - it is the theorem of Schmidt [22].

Theorem 2. The following estimate is valid for all collections $\mathscr{P}_{N} \subset[0,1]^{2}$ :

$$
\left\|D_{N}\right\|_{\infty} \gtrsim \log N
$$

This is larger than Roth's $L^{2}$ bound by $\sqrt{\log N}$. The difference between the two estimates points to the fact that for extremal choices of sets $\mathscr{P}_{N}$, the $L^{\infty}$ norm of $D_{N}$ is obtained on a set so small it cannot be seen on the scale of $L^{p}$ spaces. We will return to this point below.

In dimensions 3 and higher partial results began with a breakthrough work of J. Beck [1] in dimension $d=3$. The following result is due to Bilyk and Lacey [5] in dimension $d=3$, and Bilyk, Lacey, Vagharshakyan [6] in dimensions $d \geq 4$.

Theorem 3. In dimensions $d \geq 3$ there exists $\eta=\eta(d) \geq c / d^{2}$ for which the following estimate holds for all collections $\mathscr{P}_{N} \subset[0,1]^{d}$ :

$$
\left\|D_{N}\right\|_{\infty} \gtrsim(\log N)^{\frac{d-1}{2}+\eta} .
$$


This is larger than Roth's bound by $(\log N)^{\eta}$. Beck's original result in dimension $d=3$ had a much smaller doubly logarithmic term $(\log \log N)^{\frac{1}{8}-\varepsilon}$ in place of $(\log N)^{\eta}$. The proof strategy begins with the fundamental orthogonal function method of Roth and Schmidt, which we turn to in the next section. In $\sqrt{3}$ we turn to a closely related combinatorial inequality for "hyperbolic" sums of multiparameter Haar functions. It serves as the core question which has related the progress on lower bounds for the discrepancy function to questions in probability and approximation theory. Based upon this inequality, it is natural to conjecture that the optimal form of the $L^{\infty}$ estimate is

Conjecture 1. In dimensions $d \geq 3$ there holds $\left\|D_{N}\right\|_{\infty} \gtrsim(\log N)^{d / 2}$.

We should mention that at the present time there is no consensus among the experts about the sharp form of the conjecture (in fact, a great number of specialist believes that $\left\|D_{N}\right\|_{\infty} \gtrsim(\log N)^{d-1}$ is the optimal bound, which is supported by the best known examples). However, in this paper we shall advocate our belief in Conjecture 1 by comparing it to other sharp conjectures in various fields of mathematics. In particular, the sharpness of Conjecture 2 in $\$ 3$ suggests that the estimate above is the best that could be obtained by the orthogonal function techniques.

The reader can consult the papers [5, 6], as well as the surveys of the first author [3, 4] for more detailed information. This research is supported in part by NSF grants DMS 1101519, 1260516 (Dmitriy Bilyk), DMS 0968499, and a grant from the Simons Foundation \#229596 (Michael Lacey).

\section{The Orthogonal Function Method}

All progress on these universal lower bounds has been based upon the orthogonal function method, initiated by Roth, with the modifications of Schmidt, as presented here. Denote the family of all dyadic intervals $I \subset[0,1]$ by $\mathscr{D}$. Each dyadic interval $I$ is the union of two dyadic intervals $I_{-}$and $I_{+}$, each of exactly half the length of $I$, representing the left and right halves of $I$ respectively. Define the Haar function associated to $I$ by $h_{I}=-\chi_{I_{-}}+\chi_{I_{+}}$. Here and throughout we will use the $L^{\infty}$ (rather than $L^{2}$ ) normalization of the Haar functions.

In dimension $d$, the $d$-fold product $\mathscr{D}^{d}$ is the collection of dyadic intervals in $[0,1]^{d}$. Given $R=R_{1} \times \cdots \times R_{d} \in \mathscr{D}^{d}$, the Haar function associated with $R$ is the tensor product

$$
h_{R}\left(x_{1}, \ldots, x_{d}\right)=\prod_{j=1}^{d} h_{R_{j}}\left(x_{j}\right) .
$$

These functions are pairwise orthogonal as $R \in \mathscr{D}^{d}$ varies.

For a $d$-dimensional vector $r=\left(r_{1}, \ldots, r_{d}\right)$ with non-negative integer coordinates let $\mathscr{D}_{r}$ be the set of those $R \in \mathscr{D}^{d}$ that for each coordinate $1 \leq j \leq d$, we have $\left|R_{j}\right|=2^{-r_{j}}$. These rectangles partition $[0,1]^{d}$. We call $f_{r}$ an $r$-function (a generalized Rademacher function) if for some choice of signs $\left\{\varepsilon_{R}: R \in \mathscr{D}_{r}\right\}$, we have 


$$
f_{r}(x)=\sum_{R \in \mathscr{D} r} \varepsilon_{R} h_{R}(x) .
$$

The following is the crucial lemma of the method. Given an integer $N$, we set $n=\left\lceil 1+\log _{2} N\right\rceil$, where $\lceil x\rceil$ denotes the smallest integer greater than or equal to $x$.

Lemma 1. In all dimensions $d \geq 2$ there is a constant $c_{d}>0$ such that for each $r$ with $|r|:=\sum_{j=1}^{d} r_{j}=n$, there is an $r$-function $f_{r}$ with $\left\langle D_{N}, f_{r}\right\rangle \geq c_{d}$. Moreover, for all $r$-functions there holds $\left|\left\langle D_{N}, f_{r}\right\rangle\right| \lesssim N 2^{-|r|}$.

The proof of the lemma is straightforward, see e.g. [21, 23, 3]. With this lemma at hand, the proof of Roth's Theorem in $L^{2}$ is as follows. Note that the requirement that $|r|=n$ says that the coordinates of $r$ must partition $n$ into $d$ parts. It follows that the number of ways to select the coordinates of $r$ is bounded above and below by a multiple of $n^{d-1}$, agreeing with the simple logic that there are $d-1$ "free" parameters: $d$ dimensions minus the restriction $|r|=n$. Set $F_{d}=\sum_{r:|r|=n} f_{r}$. Orthogonality implies that $\left\|F_{d}\right\|_{2} \lesssim n^{(d-1) / 2}$. Hence, by Cauchy-Schwarz

$$
\begin{aligned}
n^{d-1} & \lesssim \sum_{r:|r|=n}\left\langle D_{N}, f_{r}\right\rangle=\left\langle D_{N}, F_{d}\right\rangle \\
& \leq\left\|D_{N}\right\|_{2} \cdot\left\|F_{d}\right\|_{2} \leq\left\|D_{N}\right\|_{2} \cdot n^{(d-1) / 2} .
\end{aligned}
$$

The universal lower bound $n^{(d-1) / 2} \lesssim\left\|D_{N}\right\|_{2}$ follows.

Deeper properties of the discrepancy function may be deduced from finer properties of $r$-functions. A key property is the classical Littlewood-Paley inequality for Haar functions:

Theorem 4. For $p \geq 2$, we have the inequality

$$
\left\|\sum_{I \in \mathscr{D}} \alpha_{I} h_{I}\right\|_{p} \leq C \sqrt{p}\left\|\left[\sum_{I \in \mathscr{D}} \frac{\left|\alpha_{I}\right|^{2}}{|I|^{2}} \chi_{I}\right]^{1 / 2}\right\|_{p}
$$

where $C$ is an absolute constant, and the coefficients $\alpha_{I}$ take values in a Hilbert space $\mathbf{H}$.

The right-hand side is the Littlewood-Paley (martingale) square function of the left hand side. This inequality can be viewed as an extension of orthogonality and Parseval's identity to values of $p$ other than 2, and it is often useful to keep track of the growth of $L^{p}$ norms. The fact that one can allow Hilbert space value coefficients permits repeated application of the inequality. The role of the Hilbert space valued coefficients is the focus of [14], which includes more information about multiparameter harmonic analysis, relevant to this subject.

Consider the dual function in (3), $F_{d}=\sum_{r:|r|=n} f_{r}$. As discussed earlier, the index set $\{r:|r|=n\}$ has $d-1$ free parameters. The function $F_{d}$ is a Haar series in the first variable, so the inequality (4) applies. On the right-hand side, the square function can be viewed as an $\ell^{2}$-valued Haar series in the second variable, hence (4) applies again, see [6, 3] for details. Continuing this $d-1$ times, one arrives at 


$$
\left\|F_{d}\right\|_{p} \lesssim p^{(d-1) / 2} n^{(d-1) / 2}, \quad 2 \leq p<\infty .
$$

Repeating (3) verbatim (with Hölder replacing Cauchy-Schwarz), one obtains $n^{(d-1) / 2} \lesssim\left\|D_{N}\right\|_{q}$ for $1<q<2$.

If one is interested in endpoint estimates, it is useful to rephrase the inequalities for $F_{d}$ above in the language of Orlicz spaces. For a convex increasing function $\psi$ : $\mathbb{R}_{+} \rightarrow \mathbb{R}_{+}$with $\psi(0)=0$, the Orlicz space $L^{\psi}$ is defined as the space of measurable functions $f:[0,1]^{d} \rightarrow \mathbb{R}$ for which

$$
\|f\|_{L^{\psi}}=\inf \left\{K>0: \int_{[0,1]^{d}} \psi(|f(x)| / K) d x \leq 1\right\} .
$$

In particular, for $\psi(t)=t^{p}$ one obtains the standard $L^{p}$ spaces, while $\exp \left(L^{\alpha}\right)$ and $L(\log L)^{\beta}$ denote Orlicz spaces generated by functions equal to $e^{t^{\alpha}}$ and $t \log ^{\beta} t$ respectively, when $t$ is large enough. These spaces serve as refinements of the endpoints of the $L^{p}$ scale, as for each $1<p<\infty, \alpha, \beta>0$ we have the embeddings $L^{\infty} \subset \exp \left(L^{\alpha}\right) \subset L^{p}$ and $L^{p} \subset L(\log L)^{\beta} \subset L^{1}$.

The polynomial growth in the $L^{p}$ norms of $F_{d}(5)$ translates into exponential integrability estimates, namely $\left\|F_{d}\right\|_{\exp \left(L^{2 /(d-1)}\right)} \lesssim n^{(d-1) / 2}$, since

$$
\|f\|_{\exp \left(L^{\alpha}\right)} \simeq \sup _{p>1} p^{-1 / \alpha}\|f\|_{p}, \quad \alpha>0 .
$$

The dual space to $\exp \left(L^{2 /(d-1)}\right)$ is $L(\log L)^{(d-1) / 2}$, hence we see that

$$
n^{(d-1) / 2} \lesssim\left\|D_{N}\right\|_{L(\log L)(d-1) / 2} .
$$

A well-known result of Halász [15] is a ' $\sqrt{\log L}$ ' improvement of this estimate in dimension $d=2$. Indeed, we have the following theorem valid for all dimensions, see [18].

Theorem 5. For dimensions $d \geq 2$, there holds $\left\|D_{N}\right\|_{L(\log L)^{(d-2) / 2}} \gtrsim(\log N)^{(d-1) / 2}$.

Notice that for $d=2$ one recovers Halász's $L^{1}$ bound

$$
\sqrt{\log N} \lesssim\left\|D_{N}\right\|_{1}
$$

In dimension $d=2$, the argument of Halász can be rephrased into the estimate

$$
\sqrt{n} \lesssim\left\langle D_{N}, \sin \left(\frac{c}{\sqrt{n}} F_{2}\right)\right\rangle, \quad 0<c<1 \text { sufficiently small. }
$$

This immediately shows that $\left\|D_{N}\right\|_{1} \gtrsim \sqrt{n}$ in dimension $d=2$. There is a relevant endpoint estimate of the Littlewood-Paley inequalities, namely the Chang-WilsonWolff inequality [10]. Employing extensions of this inequality and the estimate above, one can give a proof of Theorem 5 in dimensions $d \geq 3$.

It is a well-known conjecture that in all dimensions $d \geq 3$ one has the estimate 


$$
\left\|D_{N}\right\|_{1} \gtrsim(\log N)^{(d-1) / 2}
$$

on the $L^{1}$ norm of the discrepancy function. Any improvement of Theorem 5 would yield progress on this conjecture.

\section{The Small Ball Inequality}

Lower bounds on the discrepancy function are related through proof techniques to subjects in different areas of mathematics. They include, in particular, the so-called small deviation inequalities for the Brownian sheet in probability theory, complexity bounds for certain Sobolev spaces in approximation theory, and a combinatorial inequality involving multivariate Haar functions in the unit cube. We refer the reader to the references [5, 6, 3, 4] for more information, and emphasize that the questions in probability and approximation theory are parts of very broad areas of investigation with additional points of contact with discrepancy theory and many variations of the underlying themes.

According to the idea introduced in the previous section, the behavior of $D_{N}$ is essentially defined by its projection onto the span of $\left\{h_{R}: R \in \mathscr{D}^{d},|R|=2^{-n}\right\}$. It is therefore reasonable to model the discrepancy bounds by estimates of the linear combinations of such Haar functions (we call such sums "hyperbolic"). The problem of obtaining lower bounds for sums of Haar functions supported by rectangles of fixed volume - known as the Small Ball inequality - arises naturally in the aforementioned problems in probability and approximation theory. While in the latter fields versions of this inequality have important formal implications, its connection to discrepancy estimates is still only intuitive and is not fully understood. However, most known proof methods are easily transferred from one problem to another. The conjectured form of the inequality is the following.

Conjecture 2. [The Small Ball Conjecture] For dimension $d \geq 3$ we have the inequality

$$
2^{-n} \sum_{|R|=2^{-n}}\left|\alpha_{R}\right| \lesssim n^{(d-2) / 2}\left\|_{R \in \mathscr{D} d:|R|=2^{-n}} \alpha_{R} h_{R}\right\|_{\infty}
$$

valid for all real-valued coefficients $\alpha_{R}$.

The subject of the conjecture is the exact exponent of $n$ the right-hand side. This conjecture is better, by one square root of $n$, than a trivial estimate available from the Cauchy-Schwartz inequality. Indeed, with $n^{(d-2) / 2}$ replaced by $n^{(d-1) / 2}$ it holds for the $L^{2}$ norm: 


$$
\begin{aligned}
\left\|\sum_{R \in \mathscr{D}^{d}:|R|=2^{-n}} \alpha_{R} h_{R}\right\|_{2} & =\left(\sum_{|R|=2^{-n}}\left|\alpha_{R}\right|^{2} 2^{-n}\right)^{\frac{1}{2}} \\
& \gtrsim \frac{\sum_{|R|=2^{-n}}\left|\alpha_{R}\right| 2^{-n / 2}}{\left(n^{d-1} 2^{n}\right)^{\frac{1}{2}}}=n^{-\frac{d-1}{2}} \cdot 2^{-n} \sum_{|R|=2^{-n}}\left|\alpha_{R}\right|,
\end{aligned}
$$

where we have used the fact that the total number of rectangles $R \in \mathscr{D}^{d}$ is $\approx n^{d-1} 2^{n}$. This computation is similar in spirit to (3) establishing Roth's $L^{2}$ discrepancy bound. Generally, the Small Ball Conjecture bears a strong resemblance to Conjecture 1 about the discrepancy function. Indeed, in both cases one gains a square root of the logarithm over the $L^{2}$ bound.

One can consider a restricted version of inequality (8), which appears to contain virtually all the complexity of the general inequality and is sufficient for applications:

$$
\left\|\sum_{|R|=2^{-n}} \varepsilon_{R} h_{R}\right\|_{\infty} \gtrsim n^{d / 2}, \quad \varepsilon_{R} \in\{-1,0,1\}
$$

subject to the requirement that $\sum_{|R|=2^{-n}}\left|\varepsilon_{R}\right| \geq c 2^{n} n^{d-1}$ for a fixed small constant $c>0$, in other words, at least a fixed proportion of the coefficients $\varepsilon_{R}$ are non-zero. The relation to the discrepancy estimates becomes even more apparent for this form of the inequality. For instance, the trivial bound (9) becomes

$$
\left\|\sum_{|R|=2^{-n}} \varepsilon_{R} h_{R}\right\|_{\infty} \geq\left\|\sum_{|R|=2^{-n}} \varepsilon_{R} h_{R}\right\|_{2} \gtrsim n^{(d-1) / 2} .
$$

Compare this to Roth's bound (1), and compare (10) to Conjecture 1, The similarities between the discrepancy estimates and the Small Ball inequality are summarized in Table 1

A more restrictive version of inequality $(8)$ with $\varepsilon_{R}= \pm 1$ (the signed small ball inequality) does allow for some proof simplifications, but has no direct consequences. The papers [7, 6] study this restricted inequality, using only the fundamental inequality - Lemma 2 of 6 This case will likely continue to be a proving ground for new techniques in this problem.

Conjecture 2 is sharp: for independent random selection of coefficients (either random signs or Gaussians), the supremum is at most $\mathrm{Cn}^{d / 2}$,

$$
\mathbb{E}\left\|\sum_{|R|=2^{-n}} \alpha_{R} h_{R}\right\|_{\infty} \simeq n^{d / 2}
$$

Unfortunately, random selection of coefficients does not seem to be a guide to the sums that are hardest to analyze. The sharpness of the Small Ball Conjecture justifies our belief in the optimality of Conjecture 1 in discrepancy theory. 


\begin{tabular}{|c|c|}
\hline Discrepancy estimates & Small Ball inequality (signed) \\
\hline \multicolumn{2}{|c|}{ Dimension $d=2$} \\
\hline $\begin{array}{c}\left\|D_{N}\right\|_{\infty} \gtrsim \log N \\
\text { (Schmidt, '72; Halász, ' } 81 \text { ) }\end{array}$ & $\begin{array}{l}\left\|\sum_{|R|=2^{-n}} \varepsilon_{R} h_{R}\right\|_{\infty} \gtrsim n \\
\text { (Talagrand, '94; Temlyakov, '95) }\end{array}$ \\
\hline \multicolumn{2}{|c|}{ Higher dimensions, $L^{2}$ bounds } \\
\hline$\left\|D_{N}\right\|_{2} \gtrsim(\log N)^{(d-1) / 2}$ & $\left\|\sum_{|R|=2^{-n}} \varepsilon_{R} h_{R}\right\|_{2} \gtrsim n^{(d-1) / 2}$ \\
\hline \multicolumn{2}{|c|}{ Higher dimensions, conjecture } \\
\hline$\left\|D_{N}\right\|_{\infty} \gtrsim(\log N)^{d / 2}$ & $\left\|\sum_{|R|=2^{-n}} \varepsilon_{R} h_{R}\right\|_{\infty} \gtrsim n^{d / 2}$ \\
\hline \multicolumn{2}{|c|}{ Higher dimensions, known results } \\
\hline$\left\|D_{N}\right\|_{\infty} \gtrsim(\log N)^{\frac{d-1}{2}+\eta}$ & $\left\|\sum_{|R|=2^{-n}} \varepsilon_{R} h_{R}\right\|_{\infty} \gtrsim n^{\frac{d-1}{2}+\eta}$ \\
\hline
\end{tabular}

Table 1 Discrepancy estimates and the signed Small Ball inequality

\section{Connections to Probability and Approximation Theory}

We briefly touch upon the connections of the Small Ball inequality (8) to problems in other fields. A very detailed account of these relations is contained in [4].

\subsection{Approximation theory: Metric entropy of classes with dominating mixed smoothness.}

Let $M W^{p}$ be the image of the unit ball $L^{p}\left([0,1]^{d}\right)$ under the integration operator $(\mathscr{T} f)(x)=\int_{0}^{x_{1}} \ldots \int_{0}^{x_{d}} f(y) d y$, i.e. in some sense $M W^{p}$ is the set of functions on $[0,1]^{d}$ whose mixed derivative $\frac{\partial^{d} f}{\partial x_{1} \partial x_{2} \ldots \partial x_{d}}$ has $L^{p}$ norm bounded by one. This set is compact in the $L^{\infty}$ metric and its compactness may be measured by covering numbers. Let $N(\varepsilon, p, d)$ be the cardinality of the smallest $\varepsilon$-net of $M W^{p}$ in the $L^{\infty}$ norm. The exact asymptotics of these numbers as $\varepsilon \downarrow 0$ is a subject of conjecture.

Conjecture 3. For $d \geq 2$, we have $\log N(\varepsilon, 2, d) \simeq \varepsilon^{-1}(\log 1 / \varepsilon)^{d-1 / 2}$, as $\varepsilon \downarrow 0$.

The case $d=2$ is settled [26], and the upper bound is known in all dimensions [13]. Inequalities similar to the Small Ball Conjecture (8) lead to lower bounds on the covering numbers. 


\subsection{Probability: The small ball problem for the Brownian sheet.}

Consider the Brownian sheet $B_{d}$, i.e. a centered multiparameter Gaussian process characterized by the covariance relation $\mathbb{E} B_{d}(s) \cdot B_{d}(t)=\prod_{j=1}^{d} \min \left\{s_{j}, t_{j}\right\}$. The problem deals with the precise behavior of $\mathbb{P}\left(\|B\|_{C\left([0,1]^{d}\right)}<\varepsilon\right)$, the small deviation (or small ball) probabilities of $B_{d}$.

There is an exciting formal equivalence established by Kuelbs and Li [16, 17] between the small ball probabilities and the metric entropy of the unit ball of the reproducing kernel Hilbert space, which in the case of the Brownian sheet is $W M^{2}$. This yields an equivalent conjecture:

Conjecture 4. In dimensions $d \geq 2$, for the Brownian sheet $B$ we have

$$
-\log \mathbb{P}\left(\|B\|_{C\left([0,1]^{d}\right)}<\varepsilon\right) \simeq \varepsilon^{-2}(\log 1 / \varepsilon)^{2 d-1}, \quad \varepsilon \downarrow 0 .
$$

The upper bounds are known for $d \geq 2$ [13], while the lower bound for $d=2$ has been obtained by Talagrand [26] using (8). It is worth mentioning that Conjecture 4 explains the nomenclature small ball inequality.

\subsection{Summary of the connections}

The connections between the Small Ball Conjecture and these problems is illustrated in Figure 1. Solid arrows represent known formal implications, while a dashed line denotes an informal heuristic relation. Hopefully, other lines, as well as other nodes, will be added to this diagram in the future. In particular, we expect that the theory of empirical processes may connect the discrepancy bounds to the small deviation probabilities.

\section{Riesz Product Techniques}

The only case in which the Small Ball inequality (8) is known in full generality is dimension $d=2$, which was proved by M. Talagrand [26].

Theorem 6. In dimension $d=2$, there holds for all $n$,

$$
2^{-n} \sum_{|R|=2^{-n}}\left|\alpha_{R}\right| \lesssim\left\|\sum_{|R| \geq 2^{-n}} \alpha_{R} h_{R}\right\|_{\infty} .
$$

Soon after M. Talagrand proved Conjecture 2 in dimension $d=2$, V. Temlyakov [27] has given an alternative elegant proof of this inequality, which strongly resonated with the argument of Halász [15] for (2). We shall present this technically 


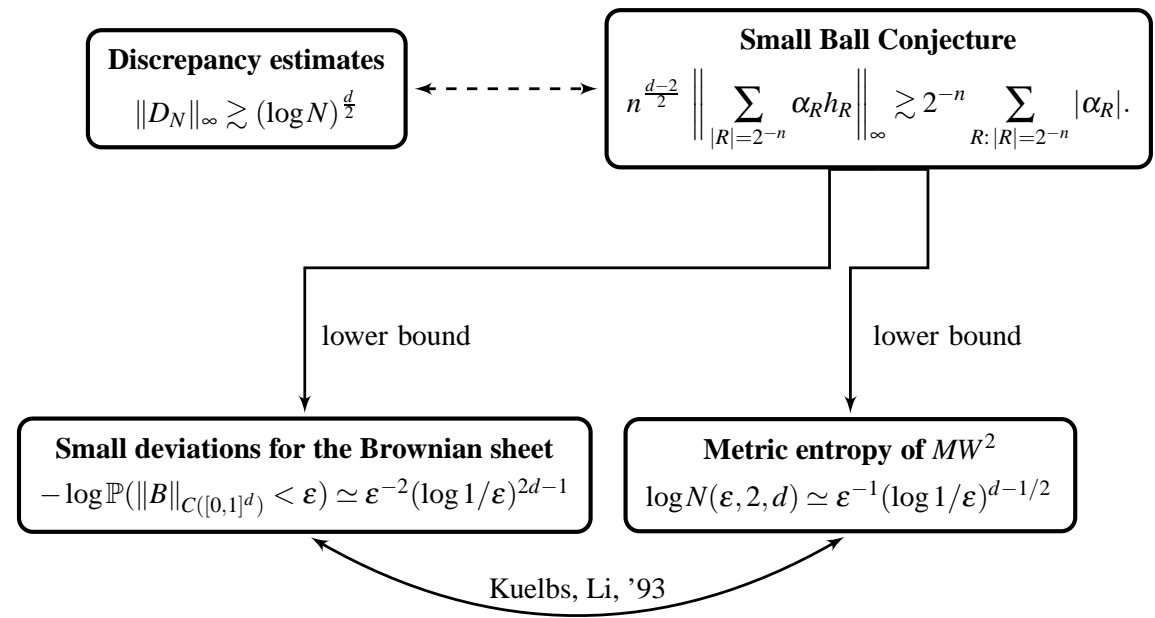

Fig. 1 Connections between the Small Ball Conjecture and other problems

simpler proof and then explain the adjustments needed to obtain the discrepancy bound.

All the endpoint estimates in dimension $d=2$ are based upon a very special property of the two-dimensional Haar functions and the associated $r$-functions, product rule: if $R, R^{\prime} \in \mathscr{D}^{2}$ are not disjoint, $R \neq R^{\prime}$, and $|R|=\left|R^{\prime}\right|$, then

$$
h_{R} \cdot h_{R^{\prime}}= \pm h_{R \cap R^{\prime}}
$$

i.e. the product of two Haar functions is again Haar, or equivalently, if $|r|=|s|=n$, then the product $f_{r} \cdot f_{s}=f_{t}$ is also an $r$ function, where $t=\left(\min \left\{r_{1}, s_{1}\right\}, \min \left\{r_{2}, s_{2}\right\}\right)$. In higher dimensions two different boxes of the same volume may coincide in one of the coordinates, in which case $h_{R_{k}} \cdot h_{R_{k}^{\prime}}=h_{R_{k}}^{2}=\mathbf{1}_{R_{k}}$. This loss of orthogonality leads to major complications in dimensions three and above.

Proof. For each $j=0, \ldots, n$ consider the $r$-functions $f_{(j, n-j)}=\sum_{\substack{|R|=2^{-n} \\\left|R_{1}\right|=2^{-j}}} \operatorname{sgn}\left(\alpha_{R}\right) h_{R}$.

In dimension $d=2$ the summation conditions uniquely define the shape of a dyadic rectangle. The product rule drives this argument. We construct the following Riesz product

$$
\Psi:=\prod_{j=1}^{n}\left(1+f_{(j, n-j)}\right)=1+\sum_{R \in \mathscr{D} d:|R|=2^{-n}} \operatorname{sgn}\left(\alpha_{R}\right) h_{R}+\Psi_{>n},
$$

where, by the product rule, $\Psi_{>n}$ is a linear combination of Haar functions supported by rectangles of area less than $2^{-n}$, and make three simple observations

(i) $\Psi \geq 0$, since each factor is either 0 or 2 . 
(ii) Next, $\int \Psi(x) d x=1$. Indeed, expand the product in (11) - the initial term is 1 , while all the higher-order terms are Haar functions with mean zero.

(iii) Therefore $\Psi$ has $L^{1}$ norm 1: $\|\Psi\|_{1}=1$.

By the same token, using orthogonality,

$$
\left\|\sum_{|R|=2^{-n}} \alpha_{R} h_{R}\right\|_{\infty} \geq\left\langle\sum_{|R|=2^{-n}} \alpha_{R} h_{R}, \Psi\right\rangle=2^{-n} \cdot \sum_{|R|=2^{-n}}\left|\alpha_{R}\right|
$$

since $\left\langle h_{R}, h_{R}\right\rangle=2^{-n}$.

Rather than proving Schmidt's discrepancy lower bound, we shall explain how the above argument could be adapted to obtain Halász's proof of (2). These are the necessary changes:

- Building blocks: Instead of the $r$-functions $f_{(j, n-j)}=\sum \operatorname{sgn}\left(\alpha_{R}\right) h_{R}$ used above, we take the $r$-functions provided by Lemma 1 with the property that $\left\langle D_{N}, f_{r}\right\rangle \gtrsim 1$.

- Riesz product: The test function $\Psi:=\prod_{j=1}^{n}\left(1+f_{(j, n-j)}\right)$ should be replaced by a slightly more complicated $\Phi=\prod_{j=1}^{n}\left(1+\gamma f_{(j, n-j)}\right)-1$, where $\gamma>0$ is a small constant.

These adjustments play the following roles: -1 in the end forces the "zero-order" term $\int D_{N}(x) d x$ to disappear, while a suitable choice of the small constant $\gamma$ takes care of the "higher-order" terms and ensures that their contribution is small. Otherwise, the proof of (2) is verbatim the same as the proof of the two-dimensional Small Ball Conjecture; the details can be found in [15, 19, 6, 3] etc. The Small Ball Conjecture may therefore be viewed as a linear term in the discrepancy estimates. These same comments apply to the proof of the $L^{1}$ estimate (7) of Halász.

The power of the Riesz product approach in discrepancy problems and the Small Ball Conjecture can be intuitively justified. The maximal values of the discrepancy function (as well as of hyperbolic Haar sums) are achieved on a very sparse, fractal set. Riesz products are known to capture such sets extremely well. In fact, $\Psi=$ $2^{n+1} \mathbf{1}_{E}$, where $E$ is the set on which all the functions $f_{k}$ are positive, i.e. $\Psi$ defines a uniform measure on the set where the $L^{\infty}$ norm is achieved. In particular, $E$ is essentially the low-discrepancy van der Corput set [3] if all $\varepsilon_{R}=1$ (in this case, $f_{(k, n-k)}$ are Rademacher functions).

Historically, Riesz products were designed to work with lacunary Fourier series, see e.g. [28], [20], [24], [25]), that is, Fourier series with harmonics supported on lacunary sequences $\left\{n_{k}\right\}$ with $n_{k+1} / n_{k}>\lambda>1$, e.g., $n_{k}=2^{k}$. The terms of such series behave like independent random variables, which resembles our situation, since the functions $f_{(j, n-j)}$ are actually independent. The failure of the product rule explains the loss of independence in higher dimensions (see [9] for this approach towards the conjecture). The strong similarity of the two-dimensional Small Ball inequality and Sidon's theorem on lacunary Fourier series [24] 


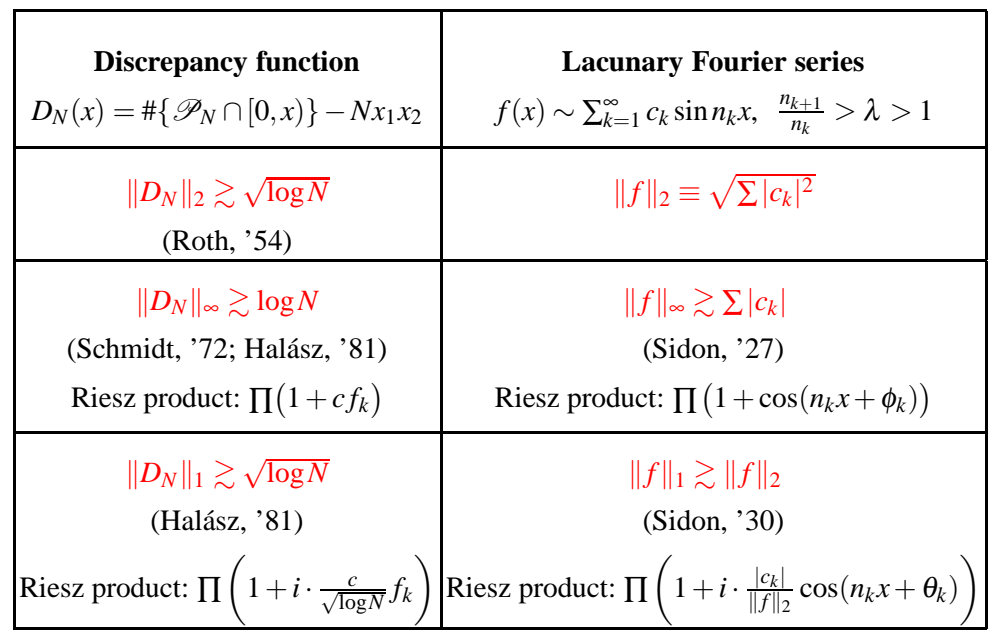

Table 2 Discrepancy function and lacunary Fourier series

$$
\left\|\sum_{|R|=2^{-n}} \alpha_{R} h_{R}\right\|_{\infty} \gtrsim 2^{-n} \sum_{R:|R|=2^{-n}}\left|\alpha_{R}\right| \quad \text { vs. } \quad\left\|\sum_{k} c_{k} \sin n_{k} x\right\|_{\infty} \gtrsim \sum_{k}\left|c_{k}\right|
$$

may be explained heuristically: the condition $|R|=2^{-n}$ effectively leaves only one free parameter, and the supports of Haar functions are dyadic - thus we obtain a oneparameter system with lacunary frequencies. The similarities between discrepancy estimates, lacunary Fourier series, and the corresponding Riesz product techniques are shown in Table 2 .

\section{Recent Results}

An improvement of the Small Ball inequality in higher dimensions has been obtained by Bilyk, Lacey, and Vagharshakyan [5, 6].

Theorem 7. For all dimensions $d \geq 3$, there is an $\eta=\eta(d)>c / d^{2}$ so that for all integers $n$ there holds

$$
2^{-n} \sum_{|R|=2^{-n}}\left|\alpha_{R}\right| \lesssim n^{\frac{d-1}{2}-\eta}\left\|\sum_{|R| \geq 2^{-n}} \alpha_{R} h_{R}\right\|_{\infty}
$$

We shall briefly explain some ideas and complications that arise in the higherdimensional case.

All simple approaches to these questions are blocked by the dramatic failure of the product rule in dimensions $d \geq 3$. This failure, as well as potential remedies, was first addressed in the breakthrough paper of József Beck [1]. Recall that the product 
rule breaks when some sides of the dyadic rectangles coincide. There is a whole range of inequalities which partially compensate for the absence of the product rule and the presence of coincidences. The simplest of these inequalities is the so-called Beck gain.

Lemma 2 (Beck gain). In dimensions $d \geq 3$ there holds

$$
\left\|\sum_{\substack{r \neq s \\|r|=|=| s \mid=n \\ r_{1}=s_{1}}} f_{r} \cdot f_{s}\right\|_{p} \lesssim p^{d-1} n^{\frac{2 d-3}{2}}, \quad 1<p<\infty .
$$

The meaning of this bound can be made clear by simple parameter counting. The summation conditions $|r|=|s|=n$ and $r_{1}=s_{1}$ "freeze" 3 parameters. Thus the pair of vectors $r$ and $s$ has $2 d-3$ free parameters, and the estimate says that they behave in an orthogonal fashion, nearly as if we had just applied the LittlewoodPaley inequality $2 d-3$ times. The actual proof is more complicated, of course, since the variables in the sum are not free as they are in (5). The paper of Beck [1] contains a weaker version of the lemma above in the case of $d=3, p=2$. The $L^{p}$ version is far more useful: the case $d=3$ is in [5], and an induction on dimension argument [6] proves the general case.

To apply the Riesz product techniques one has to be able to deal with longer, more complicated patterns of coincidences. This would require inequalities of the type

$$
\left\|\sum f_{r_{1}} \cdots f_{r_{k}}\right\|_{p} \lesssim p^{\alpha M} n^{\frac{M}{2}}
$$

where the summation is extended over all $k$-tuples of $d$-dimensional integer vectors $r_{1}, \ldots, r_{k}$ with a specified configuration of coincidences and $M$ is the number of free parameters imposed by this configuration, i.e. the free parameters should still behave orthogonally even for longer coincidences. If $k=2$, this is just (12); in [6] a partial result in this direction is obtained for $k>2$.

While the breakdown of the product rule is a feature of the method, there are intrinsic issues that demonstrate that the higher-dimensional inequality is much more delicate and difficult than the case $d=2$. There is no simple closed form for the dual function in this situation. Indeed, assume that all $\left|\alpha_{R}\right|=1$. One then wants to show that the sum $\sum_{R:|R|=2^{-n}} \alpha_{R} h_{R}(x) \gtrsim n^{d / 2}$ for some values of $x$. But every $x$ is contained in many more,namely $\mathrm{cn}^{d-1} \gg n^{d / 2}$, rectangles of volume $2^{-n}$. That is, one has to identify a collection of points which capture only a very slight disbalance between the number of positive and negative summands. There doesn't seem to be any canonical way to select such a set of points in the higher-dimensional setting, let alone construct a function similar to the Riesz product (11), which would be close to uniform measure on such a set, see [9]. 


\subsection{Other Endpoint Estimates}

The Small Ball Conjecture provides supporting evidence for Conjecture 1 on the behavior of the $L^{\infty}$ norm of the discrepancy function in dimensions $d \geq 3,\left\|D_{N}\right\| \gtrsim$ $(\log N)^{d / 2}$. On the other hand, the best known examples of point sets $\mathscr{P}_{N}$ satisfy

$\left\|D_{N}\right\|_{\infty} \lesssim(\log N)^{d-1}$. However, the techniques of the orthogonal function method cannot prove anything better than the Small Ball inequality.

As we have pointed out repeatedly, the set on which $D_{N}$ achieves its $L^{\infty}$ norm is a small set. Exactly how small has been quantified in the two-dimensional setting by Bilyk, Lacey, Parissis, Vagharshakyan [8].

Theorem 8. In dimension $d=2$, for any integer $N$

(a) for any point set $\mathscr{P}_{N}$ with $\# \mathscr{P}_{N}=N$, and $2<q<\infty$, we have

$$
\left\|D_{N}\right\|_{\exp \left(L^{q}\right)} \gtrsim(\log N)^{1-1 / q} ;
$$

(b) there exists a set $\mathscr{P}_{N}$ (a shifted van der Corput set) such that for $2 \leq q<\infty$,

$$
\left\|D_{N}\right\|_{\exp \left(L^{q}\right)} \lesssim(\log N)^{1-1 / q} .
$$

This theorem is an interpolation between Roth's and Schmidt's bounds in dimension two: when $q=2$ (the subgaussian case) the estimates resembles the $L^{2}$ behavior, $\sqrt{\log N}$, while as $q$ approaches infinity, the bounds become close to the $L^{\infty}$ estimate, $\log N$.

The crucial index $q=2$ is the exact limit of Roth's Theorem: $\left\|D_{N}\right\|_{\exp \left(L^{2}\right)} \gtrsim$ $\sqrt{\log N}$ by Roth's theorem, and there is an example of $\mathscr{P}_{N}$ for which the reverse inequality holds. It is very tempting to speculate that the Orlicz space $\exp \left(L^{2}\right)$ of subgaussian functions is the sharp space in all dimensions.

Conjecture 5. For all dimensions $d$

$$
\inf _{\mathscr{P}_{N}}\left\|D_{N}\right\|_{\exp \left(L^{2}\right)} \lesssim(\log N)^{(d-1) / 2}
$$

This would imply that in the extremal case the set $\left\{x: D_{N}(x) \geq(\log N)^{d / 2}\right\}$ would have measure at most $N^{-c}$, for some positive $c$. We are of course very far from verifying such conjectures, though they can be helpful in devising potential proof strategies for the main goal - Conjecture 1.

\section{References}

1. Beck, J.: A two-dimensional van Aardenne-Ehrenfest theorem in irregularities of distribution. Compositio Math. 72, 269-339 (1989)

2. Beck, J., Chen, W. W. L.: Irregularities of distribution. Cambridge University Press, Cambridge (1987) 
3. Bilyk, D.: On Roth's orthogonal function method in discrepancy theory. Unif. Distrib. Theory 6, 143-184 (2011)

4. Bilyk, D.: Roth's Orthogonal Function Method in Discrepancy Theory and Some New Connections. In: Panorama of discrepancy theory. Springer-Verlag (to appear)

5. Bilyk, D., Lacey, M. T.: On the small ball inequality in three dimensions. Duke Math. J. 143, 81-115 (2008)

6. Bilyk, D., Lacey, M. T., Vagharshakyan, A.: On the small ball inequality in all dimensions. J. Funct. Anal. 254, 2470-2502 (2008)

7. Bilyk, D., Lacey, M. T., Vagharshakyan, A.: On the signed small ball inequality. Online J. Anal. Comb. 3, (2008)

8. Bilyk, D., Lacey, M. T., Parissis, I., Vagharshakyan, A.: Exponential squared integrability of the discrepancy function in two dimensions. Mathematika 55, 1-27 (2009)

9. Bilyk, D., Lacey, M. T., Parissis, I., Vagharshakyan, A.: A three-dimensional signed small ball inequality. In: Dependence in probability, analysis and number theory. pp. 73-87. Kendrick Press, Heber City, UT (2010)

10. Chang, S.-Y. A., Wilson, J. M., Wolff, T. H.: Some weighted norm inequalities concerning the Schrödinger operators. Comment. Math. Helv. 60, 217-246 (1985)

11. Dick, J., Pillichshammer, F.: Digital nets and sequences. Cambridge University Press, Cambridge (2010)

12. Drmota, M., Tichy, R.: Sequences, discrepancies and applications. Springer-Verlag, Berlin (1997)

13. Dunker, T., Kühn, T., Lifshits, M., Linde, W.: Metric entropy of the integration operator and small ball probabilities for the Brownian sheet. C. R. Acad. Sci. Paris Sér. I Math. 326, 347$352(1998)$

14. Fefferman, R., Pipher, J.: Multiparameter operators and sharp weighted inequalities. Amer. J. Math. 119, 337-369 (1997)

15. Halász, G.: On Roth's method in the theory of irregularities of point distributions. In: Recent progress in analytic number theory, Vol. 2. pp. 79-94. Academic Press, London (1981)

16. Kuelbs, J., Li, W. V.: Metric entropy and the small ball problem for Gaussian measures. C. R. Acad. Sci. Paris Sér. I Math. 315, 845-850 (1992)

17. Kuelbs, J., Li, W. V.: Metric entropy and the small ball problem for Gaussian measures. J. Funct. Anal. 116, 133-157 (1993)

18. Lacey, M.: On the discrepancy function in arbitrary dimension, close to $L^{1}$. Anal. Math. 34, 119-136 (2008)

19. Matoušek, J.: Geometric discrepancy. Springer-Verlag, Berlin (2010)

20. Riesz, F.: Über die Fourierkoeffizienten einer stetigen Funktion von beschränkter Schwankung. Math. Z. 2, 312-315 (1918)

21. Roth, K. F.: On irregularities of distribution. Mathematika 1, 73-79 (1954)

22. Schmidt, W. M.: Irregularities of distribution. VII. Acta Arith. 21, 45-50 (1972)

23. Schmidt, W. M.: Irregularities of distribution. X. In: Number theory and algebra. pp. 311-329. Academic Press, New York (1977)

24. Sidon, S.: Verallgemeinerung eines Satzes über die absolute Konvergenz von Fourierreihen mit Lücken. Math. Ann. 97, 675-676 (1927)

25. Sidon, S.: Ein Satz über trigonometrische Polynome mit Lücken und seine Anwendung in der Theorie der Fourier-Reihen. J. Reine Angew. Math. 163, 251-252 (1930)

26. Talagrand, M.: The small ball problem for the Brownian sheet. Ann. Probab. 22, 1331-1354 (1994)

27. Temlyakov, V. N.: An inequality for trigonometric polynomials and its application for estimating the entropy numbers. J. Complexity 11, 293-307 (1995)

28. Zygmund, A.: Trigonometric series. Vol. I, II. Cambridge University Press, Cambridge (2002) 